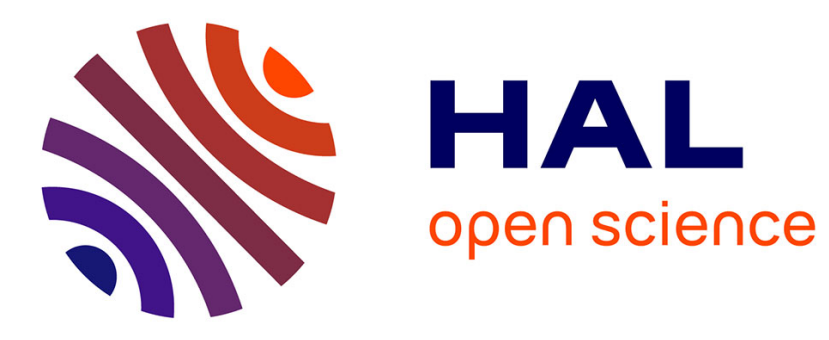

\title{
Enhanced depolarization contrast in polarization-sensitive optical coherence tomography
}

Noe Ortega-Quijano, Tahereh Marvdashti, Audrey K. Ellerbee Bowden

\section{To cite this version:}

Noe Ortega-Quijano, Tahereh Marvdashti, Audrey K. Ellerbee Bowden. Enhanced depolarization contrast in polarization-sensitive optical coherence tomography. Optics Letters, 2016, 41 (10), pp.23502353. 10.1364/OL.41.002350 . hal-01343405

\section{HAL Id: hal-01343405 \\ https://hal-univ-rennes1.archives-ouvertes.fr/hal-01343405}

Submitted on 27 Oct 2016

HAL is a multi-disciplinary open access archive for the deposit and dissemination of scientific research documents, whether they are published or not. The documents may come from teaching and research institutions in France or abroad, or from public or private research centers.
L'archive ouverte pluridisciplinaire HAL, est destinée au dépôt et à la diffusion de documents scientifiques de niveau recherche, publiés ou non, émanant des établissements d'enseignement et de recherche français ou étrangers, des laboratoires publics ou privés. 
Final Draft

\title{
Enhanced depolarization contrast in polarization-sensitive optical coherence tomography
}

Noé Ortega-Quijano ${ }^{1,2}$, Tahereh Marvdashti ${ }^{1}$ and Audrey K. Ellerbee Bowden ${ }^{1 *}$

${ }^{1}$ E.L. Ginzton Laboratory and Department of Electrical Engineering, Stanford University,

Stanford, CA 94305, USA

${ }^{2}$ Institut de Physique de Rennes, CNRS-Université de Rennes 1, Campus de Beaulieu, 35042

Rennes, France

* corresponding author

\begin{abstract}
We demonstrate the first application of the Differential Depolarization Index (DDI) for depolarization imaging in polarization-sensitive optical coherence tomography (PS-OCT). Unlike the widely used Degree of Polarization Uniformity (DOPU), the DDI is independent of the incident polarization state and, therefore, more robust to varying system and sample parameters. Moreover, it can be applied to single-input-polarization-state PS-OCT systems, and it also overcomes several limitations of the emerging depolarization index (DI) used in multiple-input-polarization-state systems. Our results on tissue phantoms and human skin prove that DDI yields significant depolarization contrast improvements compared to DOPU, which highlights its potential for depolarization imaging in PS-OCT.
\end{abstract}

Journal: Optics Letters 
Depolarization of polarized light by biological tissue is linked to tissue heterogeneity and can reveal clinically relevant features [1-4]. For example, the depolarizing properties of the retinal pigment epithelium have been used to segment layers of the retina in optical coherence tomography images $[5,6]$. While polarization-sensitive optical coherence tomography (PS-OCT) can measure the reflectivity, linear retardation and optic axis orientation of tissue layers $[7,8]$, these parameters do not directly assess the depolarization properties of a sample.

To date, the Degree of Polarization Uniformity (DOPU) [5] has been the standard metric to characterize depolarization for PS-OCT [9-12]. Additionally, a closely-related parameter has been demonstrated to detect multiple scattering [13]. Importantly, DOPU can be calculated using data from a single-input-polarization-state (SIPS) system; however, its dependence on the polarization state incident at each layer of the sample [14] leads to an inherent ambiguity in the measurement due to the dependence on illumination conditions [15]. Recently, the Depolarization Index (DI) [16] has been proposed as a more robust metric [15]; however, measurement of the DI requires multiple-input-polarization-state (MIPS) systems, which increases the measurement time and system complexity.

As an alternative to the DI, we propose that the Differential Depolarization Index (DDI) [17], which quantifies depolarization from the variance and covariance of the anisotropic parameters of the sample, is a more robust and physically meaningful method to characterize depolarization. Importantly, DDI overcomes certain inaccuracies of DI [13] and it can be used with SIPS PS-OCT systems. In this work we demonstrate that the contrast in DDI images outperforms that of DOPU 
in birefringent tissues, which highlights the superior potential of DDI for depolarization imaging in PS-OCT.

Consider a dual-channel polarization-sensitive photodetector that measures the complex signals $\tilde{A}_{H}$ and $\tilde{A}_{V}$ from PS-OCT. The corresponding Stokes vector $\overrightarrow{\mathbf{S}}$ returning from a sample is given by

$$
\overrightarrow{\mathbf{S}}=\left[\begin{array}{c}
I \\
Q \\
U \\
V
\end{array}\right]=\left[\begin{array}{c}
\tilde{A}_{H} \tilde{A}_{H}^{*}+\tilde{A}_{V} \tilde{A}_{V}^{*} \\
\tilde{A}_{H} \tilde{A}_{H}^{*}-\tilde{A}_{V} \tilde{A}_{V}^{*} \\
\tilde{A}_{H} \tilde{A}_{V}^{*}+\tilde{A}_{H}^{*} \tilde{A}_{V} \\
i\left(\tilde{A}_{H} \tilde{A}_{V}^{*}-\tilde{A}_{H}^{*} \tilde{A}_{V}\right)
\end{array}\right] .
$$

The DOPU [5], which characterizes the degree to which the polarization state of light remains uniform over a given region of tissue, is defined as

$$
\mathrm{DOPU}=\sqrt{\mu^{2}\left(Q_{\text {norm }}\right)+\mu^{2}\left(U_{\text {norm }}\right)+\mu^{2}\left(V_{\text {norm }}\right)},
$$

with $\mu(X)$ denoting the mean value of $X$ in a tissue region defined by a sliding window, and the subscript norm referring to the normalized Stokes vector that emerges after interaction with the sample. Throughout this work, we use a Gaussian sliding window of $8(\mathrm{x}) \times 8(\mathrm{z})$ pixels with a standard deviation of 1.3 pixels. The Gaussian window weighting, commonly used as an alternative to rectangular sliding windows [17], is applied to the raw Stokes vector elements before computing the DOPU using Eq. (2). The DOPU ranges from 0 for a completely scrambled state of polarization over the evaluation window to 1 for a state that is perfectly defined and constant over the window. Related to the DOPU is its complement - the Degree of Polarization Diversity (DOPD) — which is simply given by

$$
\text { DOPD }=1-\text { DOPU } .
$$


Unlike the DOPU, the DOPD yields a value of 1 for a completely depolarized state of polarization over the sliding window, leading to a more intuitive relationship with depolarization (i.e., the more depolarizing the sample is, the greater value the DOPD takes).

Because the measured Stokes vector returning from the sample corresponds to rotation on the Poincare sphere of the Stokes vector incident on the sample $[14,15]$, the value of DOPU and DOPD are intrinsically linked to the incident polarization state. In contrast with the DOPU and DOPD, the value of the DDI is directly linked to the variance and covariance of the anisotropic properties of the sample [17], which are themselves the inherent source of the depolarization [18-20]. These values may be grouped in the following covariance matrix:

$$
\Sigma=\left[\begin{array}{ccc}
\left|\sigma_{x y}\right|^{2} & \sigma_{x y, 45} & \sigma_{x y, C} \\
\sigma_{x y, 45}^{*} & \left|\sigma_{45}\right|^{2} & \sigma_{45, C} \\
\sigma_{x y, C}^{*} & \sigma_{45, C}^{*} & \left|\sigma_{C}\right|^{2}
\end{array}\right],
$$

where ${ }^{*}$ is the complex conjugate, $\left|\sigma_{i}\right|^{2}$ denotes the variance and $\sigma_{i, j}$ the covariance, with the subscripts $x y, 45$, and $C$ referring to the complex propagation constant that completely characterizes anisotropy in the $x-y, \pm 45^{\circ}$, and circular directions, respectively [21]. The DDI, denoted as $P_{\delta}$, is given by the Frobenius norm of the previous matrix: $P_{\delta}=\|\Sigma\|$. Importantly, this matrix is unchanged by the incident polarization state.

In the most general case, calculating the DDI in PS-OCT would require measuring the full Jones or Mueller-Jones matrix of the sample using a MIPS system, very much like in Mueller polarimetry [22]. However, SIPS PS-OCT systems exploit the fact that, in most cases, dichroism and circular anisotropy are negligible. Working from this assumption, a clear advantage of SIPS PS-OCT is 
that the parameters contained in the covariance matrix $\sum$ can be determined directly from known equations that solve for the linear retardance $\eta$ and optic axis orientation $\theta[7,8]$. Hence, the covariance matrix takes the simplified form

$$
\Sigma=\left[\begin{array}{ccc}
\left|\sigma\left(\eta_{x y}\right)\right|^{2} & \sigma\left(\eta_{x y}, \eta_{45}\right) & 0 \\
\sigma\left(\eta_{x y}, \eta_{45}\right) & \left|\sigma\left(\eta_{45}\right)\right|^{2} & 0 \\
0 & 0 & 0
\end{array}\right],
$$

where $\eta_{x y}$ and $\eta_{45}$ are the linear retardance along the $x-y$ and $\pm 45^{\circ}$ directions [23]:

$$
\begin{aligned}
& \eta_{x y}=\eta \cos (2 \theta) \\
& \eta_{45}=\eta \sin (2 \theta)
\end{aligned} .
$$

The variances and covariances included in Eq. (5) are calculated for the sliding window described above, where the Gaussian weighting is applied to the raw $\eta_{x y}$ and $\eta_{45}$. From these values, the Differential Depolarization Index can be calculated as

$$
P_{\delta}=\sqrt{\left|\sigma\left(\eta_{x y}\right)\right|^{4}+\left|\sigma\left(\eta_{45}\right)\right|^{4}+2\left|\sigma\left(\eta_{x y}, \eta_{45}\right)\right|^{2}} .
$$

Note that $P_{\delta}$ is 0 for a uniform sample in which the linear retardance is perfectly defined over a given sliding window and is arbitrarily large for increasingly depolarizing samples. As it is convenient to have a bounded depolarization metric for imaging purposes, we introduce the following normalization:

$$
\mathrm{DDI}=\tanh \left(P_{\delta}\right)
$$

Thus, similar to the DOPD, the DDI varies from 0 for a non-depolarizing sample to 1 for a completely polarization-scrambling one. Notice that the key difference between the two metrics is that the DDI describes the variance in the underlying anisotropic parameters of the sample, while 
the DOPD merely describes the changes in the polarization state of light that traverses the sample. In this sense, the DDI is a more accurate metric to characterize the intrinsic depolarizing properties of the sample.

Let us illustrate this by the following a case study. Consider a linearly birefringent tissue layer, whose well-known Mueller matrix is that of a linear retarder

$$
\mathbf{M}_{\mathbf{L R}}=\left[\begin{array}{cccc}
1 & 0 & 0 & 0 \\
0 & C_{\eta} S_{2 \theta}^{2}+C_{2 \theta}^{2} & \left(1-C_{\eta}\right) C_{2 \theta} S_{2 \theta} & -S_{\eta} S_{2 \theta} \\
0 & \left(1-C_{\eta}\right) C_{2 \theta} S_{2 \theta} & C_{\eta} C_{2 \theta}^{2}+S_{2 \theta}^{2} & S_{\eta} C_{2 \theta} \\
0 & S_{\eta} S_{2 \theta} & -S_{\eta} C_{2 \theta} & C_{\eta}
\end{array}\right],
$$

where the compact notation $C_{n \alpha}^{m}=\cos ^{m}(n \alpha)$ and $S_{n \alpha}^{m}=\sin ^{m}(n \alpha)$ has been used. In this example, we shall assume that the linear retardance $\eta$ is deterministic, whereas the axis orientation $\theta$ presents spatial stochastic fluctuations. Furthermore, we consider that the probability density function of the axis orientation is a Wrapped-Gaussian (WG) distribution, a convenient angular statistics model that wraps the Gaussian distribution around the unit circle [24]:

$$
f_{W G}\left(\theta ; \bar{\theta}, \sigma_{\theta}\right)=\frac{1}{\sqrt{2 \pi} \sigma_{\theta}} \sum_{k=-\infty}^{+\infty} \exp \left[-\frac{(\theta-\bar{\theta}+2 k \pi)^{2}}{2 \sigma_{\theta}^{2}}\right] \text {, }
$$

with $\bar{\theta}$ and $\sigma_{\theta}$, respectively, being the axis orientation mean and standard deviation. The resulting Mueller matrix $\mathbf{M}_{\mathbf{L R}}^{\Delta}$ of a spatial window over the modeled layer is the spatial average of $\mathbf{M}_{\mathbf{L R}}$ [25], where the spatial averages of the trigonometric functions are $\left\langle C_{n \theta}\right\rangle=\exp \left(-n^{2} \sigma_{\theta}^{2} / 2\right) C_{n \bar{\theta}}$ and $\left\langle S_{n \theta}\right\rangle=\exp \left(-n^{2} \sigma_{\theta}^{2} / 2\right) S_{n \bar{\theta}}$ due to the WG distribution properties [24]. 
Let us consider a SIPS PS-OCT system with three different polarization states incident onto the modeled layer: $\overrightarrow{\mathbf{S}}_{\mathbf{x}}=\left[\begin{array}{llll}1 & 1 & 0 & 0\end{array}\right]^{\mathrm{T}}, \overrightarrow{\mathbf{S}}_{45}=\left[\begin{array}{llll}1 & 0 & 1 & 0\end{array}\right]^{\mathrm{T}}$, and $\overrightarrow{\mathbf{S}}_{\mathbf{r c}}=\left[\begin{array}{llll}1 & 0 & 0 & 1\end{array}\right]^{\mathrm{T}}$. The output Stokes vector $\overrightarrow{\mathbf{S}}$ for any incident $\overrightarrow{\mathbf{S}}_{\text {in }}$ is given by $\overrightarrow{\mathbf{S}}=\mathbf{M}_{\mathbf{L R}}^{\Delta} \overrightarrow{\mathbf{S}}_{\text {in }}$. Then, the DOPU is calculated as the degree of polarization of $\overrightarrow{\mathbf{S}}$, while the DDI is obtained from the differential Mueller matrix of $\mathbf{M}_{\mathbf{L R}}^{\Delta}$ following the procedure described in [17]. The resulting DOPD and DDI for three sets of layer properties are presented in Fig. 1. The standard deviation of the axis orientation $\sigma_{\theta}$ is made to vary from $0^{\circ}$ to $90^{\circ}$ in all cases. In Fig. 1(a), the sample parameters are $\eta=30^{\circ}$ rad and $\bar{\theta}=10^{\circ}$ . The linear retardance is increased to $\eta=60^{\circ}$ for the same $\bar{\theta}=10^{\circ}$ in Fig. 1(b), and the curves in Fig. 1(c) correspond to $\eta=60^{\circ}$ and $\bar{\theta}=55^{\circ}$. Note that in all three graphs, both DDI and DOPD monotonically increase with the standard deviation $\sigma_{\theta}$, as expected, independent of the starting sample parameters. That is, the sample becomes more depolarizing as $\sigma_{\theta}$ increases. However, we observe that DOPD strongly varies as a function of the input polarization state, evidenced by the offsets of the three lines within a given subfigure corresponding to the value of DOPD for the different input states. Moreover, one observes that the value of the DOPD further depends on the linear retardance (compare DOPD $\left(\overrightarrow{\mathbf{S}}_{\mathrm{rp}}\right)$ in Figs. 1(a)-(b)) and the mean axis orientation (compare $\operatorname{DOPD}\left(\overrightarrow{\mathbf{S}}_{\mathbf{x y}}\right)$ and $\operatorname{DOPD}\left(\overrightarrow{\mathbf{S}}_{45}\right)$ in Figs. 1(b)-(c)). This is in contrast to the DDI, for which all three lines for different input polarization states overlap. Finally, recall that every SIPS PS-OCT system is prone to measurement errors when the polarization state incident on a given layer aligns with the optic axis of that layer, which requires an analysis of the "convergence loci" of the system as pointed out in [8]. This inherent limitation would equally affect DOPU and DDI, so the precision of depolarization metrics ultimately depends on the accuracy of the SIPS PS-OCT system itself. 
Note that MIPS PS-OCT systems do not share this limitation and can achieve accurate measurement of anisotropic properties for any sample; importantly, the DDI can also be appliedto MIPS systems and could further characterize depolarization due to diattenuation.

Experiments were carried out using a SIPS PS-OCT system with a configuration similar to that described in previous works [7]. The system comprised a swept-source laser (HSL-2100-ST, Santec) with a center wavelength of $1310 \mathrm{~nm}$ and axial and lateral resolutions of $4.7 \mu \mathrm{m}$ and 10 $\mu \mathrm{m}$ respectively. Measurements were performed at an A-line rate of $20 \mathrm{kHz}$. The power incident on the sample was $2.5 \mathrm{~mW}$ and the SNR of the system was $99 \mathrm{~dB}$. To quantify the difference in contrast between the DOPD and DDI images we relied on the Weber contrast parameter:

$$
C_{w}=\frac{I_{\text {strong }}-I_{\text {weak }}}{I_{\text {weak }}}
$$

where $I_{\text {strong }}$ and $I_{\text {weak }}$ are the average depolarization metric values in a strongly- and weaklydepolarizing tissue region.

To validate the capacity of the DDI to effectively detect depolarization, we measured a tissue phantom composed of several alternating layers of clear PDMS and $\mathrm{TiO}_{2}+\mathrm{PDMS}$ [26]. We expected the phantom to only show scattering and optical extinction due to the scattering coefficient, with no birefringent effects. Fig. 2(a) shows the results obtained for the phantom sample, with the reflectance given in dB. Fig. 2(b) includes the normalized DOPU (left) and DOPD (right), while the normalized DDI is presented in Fig. 2(c). All depolarization metrics clearly distinguish the scattering material from the background, which constitute a first confirmation that the DDI is appropriately detecting depolarization in the same way as DOPU and DOPD. The 
Weber contrast calculated from the mean values in the strongly- and weakly-depolarizing regions shown in Figs. 2(b) and 2(c) is 0.644 and 0.674, respectively. The small relative increase in contrast of DDI over DOPD (4.7\%) highlights the similar performance of both metrics in non-birefringent scattering samples.

To demonstrate the performance of the DDI in biological tissue, we next measured an ex-vivo human skin sample excised from a region showing a BCC (basal cell carcinoma). The sample was collected under an approved IRB protocol at Stanford University, whereby the subject gave verbal consent. The backscattered intensity image is shown in Fig. 3(a). The linear retardance along the $x-y$ and $\pm 45^{\circ}$ directions $\left(\eta_{x y}\right.$ and $\eta_{45}$, Eq. (6)) are depicted in Figs. 3(b) and 3(c), respectively. Fig. 3(d) shows the normalized DOPU (left) and DOPD (right), and the normalized DDI image is given in Fig. 3(e). Note that for visualization purposes, all images were subjected to an intensity threshold determined from the backscattered intensity image by the global image threshold Matlab function using Otsu's method [27], but the depolarization metrics and their corresponding contrast parameters were calculated from raw data prior to applying the threshold.

The strong appearance of a depolarizing structure highlights the significantly better contrast of DDI vs. DOPD. Indeed, the Weber contrast calculation for the boxed regions shown in Figs. 3(d) and 3(e) yields a relative contrast improvement of roughly $90 \%$ (0.491 for DODP vs. 0.933 for DDI). Fig. 3(f) shows the profile of the normalized DOPD and DDI taken along the dashed line shown in the depolarization images. While both DOPU and DDI detect a strongly polarizationscrambling region in the measured tissue, the depolarizing structure is much more sharply localized by DDI. Such a depolarizing tissue region is at a depth of roughly $150 \mu \mathrm{m}$ below the 
tissue surface, so it may correspond to the basal cell layer located at the epidermis and dermis junction. Melanin is produced by melanocytes in this layer, and melanin granules have been reported to depolarize light [28], so we hypothesize that the depolarization contrast in this case possibly correlates with melanin concentration, which might be increased by the presence of the BCC tumor. However, this possibility would need to be confirmed by a thoughtful study.

Fig. 4 shows the results obtained for a human nail fold. A polarization-scrambling layer in the nail plate is observable in both depolarization images, with much greater contrast apparent for DDI compared to DOPD (Figs. 4(c) and 4(d)). The contrast parameters calculated for the regions highlighted in Fig. 4 show a relative contrast increase of $84.7 \%$ in the DDI image over DOPD (0.456 for DODP vs. 0.843 for DDI). The normalized DOPD and DDI curves taken from along the dashed line are shown in Fig. 4(b). These curves confirm that both metrics are sensitive to tissue depolarization.

In summary, we have proposed an alternative method for depolarization imaging in PS-OCT based on the Differential Depolarization Index. Although generally valid for any PS-OCT system, the advantageous fact that DDI is closely related to the variance and covariance of the sample anisotropic properties enables it to be straightforwardly applied to SIPS PS-OCT. The suitability of this metric was validated on a controlled tissue phantom and subsequently applied to image depolarizing structures in human skin and nail fold samples.

Our results show that DDI yields better depolarization contrast compared to DOPU for the same polarization-scrambling structures. Moreover, the inherent independence of the DDI to incident 
polarization makes this value more convenient to measure and straightforward to interpret. Future works should consider the optimum evaluation window for DDI or investigate the potential of the DDI to aid in segmentation of PS-OCT images.

Noé Ortega-Quijano acknowledges financial support from the France-Stanford Center for Interdisciplinary Studies (Visiting Junior Scholar Fellowship). We also thank Dr. Jean Tang and Dr. Sumaira Aasi for help with collecting the tumor tissue samples.

\section{References}

1. G. Yao and L. V. Wang, Opt. Lett. 24, 537-9 (1999).

2. B. Baumann, E. Götzinger, M. Pircher, and C. K. Hitzenberger, J. Biophotonics 2, 426-34 (2009).

3. A. Pierangelo, A. Nazac, A. Benali, P. Validire, H. Cohen, T. Novikova, B. H. Ibrahim, S. Manhas, C. Fallet, M.-R. Antonelli, and A. De Martino, Opt. Express 21, 14120-30 (2013).

4. B. Baumann, J. Schirmer, S. Rauscher, S. Fialová, M. Glösmann, M. Augustin, M. Pircher, M. Gröger, and C. K. Hitzenberger, Investig. Ophthalmol. Vis. Sci. 56, 7462-72 (2015).

5. E. Götzinger, M. Pircher, W. Geitzenauer, C. Ahlers, B. Baumann, S. Michels, U. Schmidt-Erfurth, and C. K. Hitzenberger, Opt. Express 16, 16410-28 (2008).

6. T. Torzicky, M. Pircher, S. Zotter, M. Bonesi, E. Götzinger, and C. K. Hitzenberger, Opt. Express 20, 7564-74 (2012).

7. C. K. Hitzenberger, E. Götzinger, M. Sticker, M. Pircher, and A. F. Fercher, Opt. Express 9, 780-90 (2001).

8. K. L. Lurie, T. J. Moritz, and A. K. Ellerbee, Biomed. Opt. Express 3, 2273-87 (2012).

9. P. Roberts, B. Baumann, J. Lammer, B. Gerendas, J. Kroisamer, W. Bühl, M. Pircher, C. K. Hitzenberger, U. Schmidt-Erfurth, and S. Sacu, Invest. Ophthalmol. Vis. Sci. 57, 1595 (2016).

10. S. Makita, Y.-J. Hong, M. Miura, and Y. Yasuno, Opt. Lett. 39, 6783-6 (2014). 
11. B. Baumann, S. Zotter, M. Pircher, E. Götzinger, S. Rauscher, M. Glösmann, J. Lammer, U. Schmidt-Erfurth, M. Gröger, and C. K. Hitzenberger, J. Mod. Opt. 62, 1758-63 (2015).

12. M. Sugita, M. Pircher, S. Zotter, B. Baumann, K. Saito, T. Makihira, N. Tomatsu, M. Sato, and C. K. Hitzenberger, J. Biomed. Opt. 20, 016011 (2015).

13. S. G. Adie, T. R. Hillman, and D. D. Sampson, Opt. Express 15, 18033-49 (2007).

14. R. A. Chipman, Appl. Opt. 44, 2490-5 (2005).

15. N. Lippok, M. Villiger, and B. E. Bouma, Opt. Lett. 40, 3954-3957 (2015).

16. J. J. Gil and E. Bernabeu, Opt. Acta 33, 185-9 (1986).

17. N. Ortega-Quijano, F. Fanjul-Vélez, and J. L. Arce-Diego, Opt. Lett. 40, 3280-3 (2015).

18. V. Devlaminck, J. Opt. Soc. Am. A 30, 2196-204 (2013).

19. R. Ossikovski and O. Arteaga, Opt. Lett. 39, 4470-3 (2014).

20. V. Devlaminck, J. Opt. Soc. Am. A 32, 1736-43 (2015).

21. N. Ortega-Quijano and J. L. Arce-Diego, Opt. Lett. 36, 2429-31 (2011).

22. N. Ortega-Quijano and J. L. Arce-Diego, Opt. Lett. 36, 1942-4 (2011).

23. R. M. A. Azzam, J. Opt. Soc. Am. 68, 1756-67 (1978).

24. K. V. Mardia and P. E. Jupp, Directional Statistics (Wiley, 1999).

25. N. Ortega-Quijano, J. Fade, M. Roche, F. Parnet, and M. Alouini, J. Opt. Soc. Am. A 33, 434-46 (2016).

26. K. L. Lurie, G. T. Smith, S. A. Khan, J. C. Liao, and A. K. Ellerbee, J. Biomed. Opt. 19, 036009 (2014).

27. N. Otsu, IEEE Transactions on Systems, Man, and Cybernetics 9, 62-6 (1979).

28. B. Baumann, S. O. Baumann, T. Konegger, M. Pircher, E. Götzinger, F. Schlanitz, C. Schütze, H. Sattmann, M. Litschauer, U. Schmidt-Erfurth, and C. K. Hitzenberger, Biomed. Opt. Express 3, $1670-83$ (2012). 
Supplemental Information 


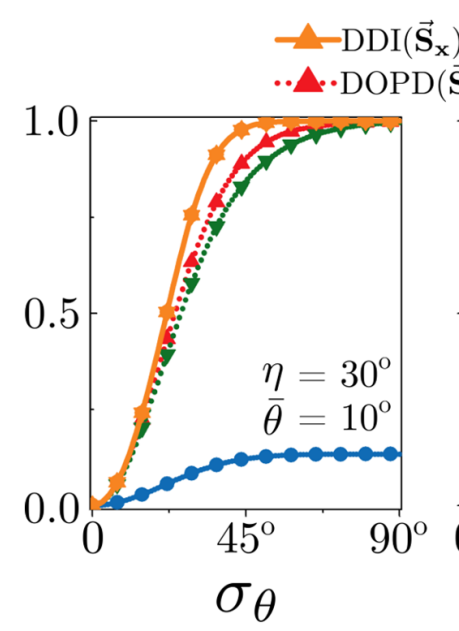

(a)

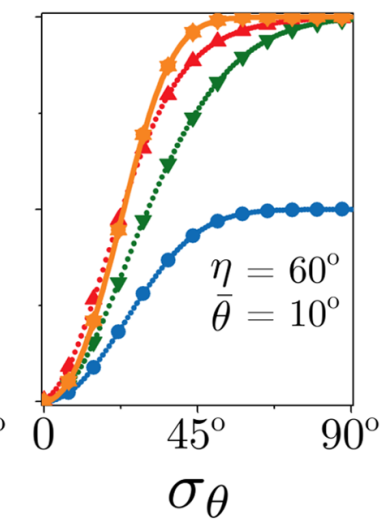

(b)
$-\mathrm{DDI}\left(\overrightarrow{\mathbf{S}}_{\mathbf{r c}}\right)$

$\cdot \operatorname{DOPD}\left(\overrightarrow{\mathbf{S}}_{\mathbf{r c}}\right)$

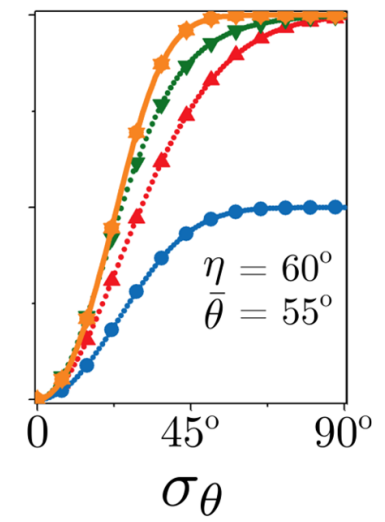

(c)

Figure 1. DDI (Differential Depolarization Index), and DOPD (Degree of Polarization Diversity; DDI=1-DOPU) for SIPS PS-OCT based on three different input polarization states $\left(\overrightarrow{\mathbf{S}}_{\mathbf{x}}:\right.$ linear along $x ; \overrightarrow{\mathbf{S}}_{45}$ : linear along $+45^{\circ}$; and $\overrightarrow{\mathbf{S}}_{\mathrm{rc}}$ : right circular). The sample properties are: (a) $\eta=30^{\circ}$ and $\bar{\theta}=10^{\circ}$; (b) $\eta=60^{\circ}$ and $\bar{\theta}=10^{\circ}$; and (c) $\eta=60^{\circ}$ and $\bar{\theta}=55^{\circ}$. The axis orientation standard deviation $\sigma_{\theta}$ is varied from $0^{\circ}$ to $90^{\circ} \mathrm{rad}$ in all cases. Note that the DDI is identical for the three input polarization states (upper curve, overlapped results), while DOPD changes (three lower curves). 


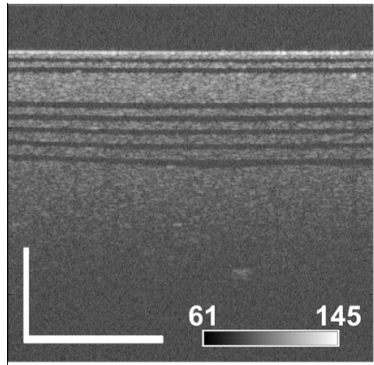

(a)

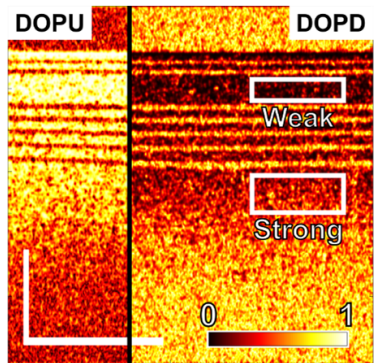

(b)

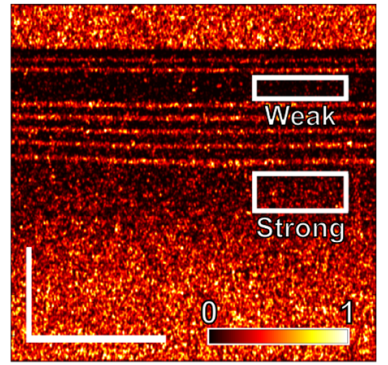

(c)

Figure 2. (a) Reflectance (in dB); (b) normalized DOPU (left) and DOPD (right); and (c)

normalized DDI. The scale bar is $0.5 \times 0.5 \mathrm{~mm}$. The weak and strong depolarization regions used for calculating contrast are shown. The relative contrast increase is $4.7 \%$.
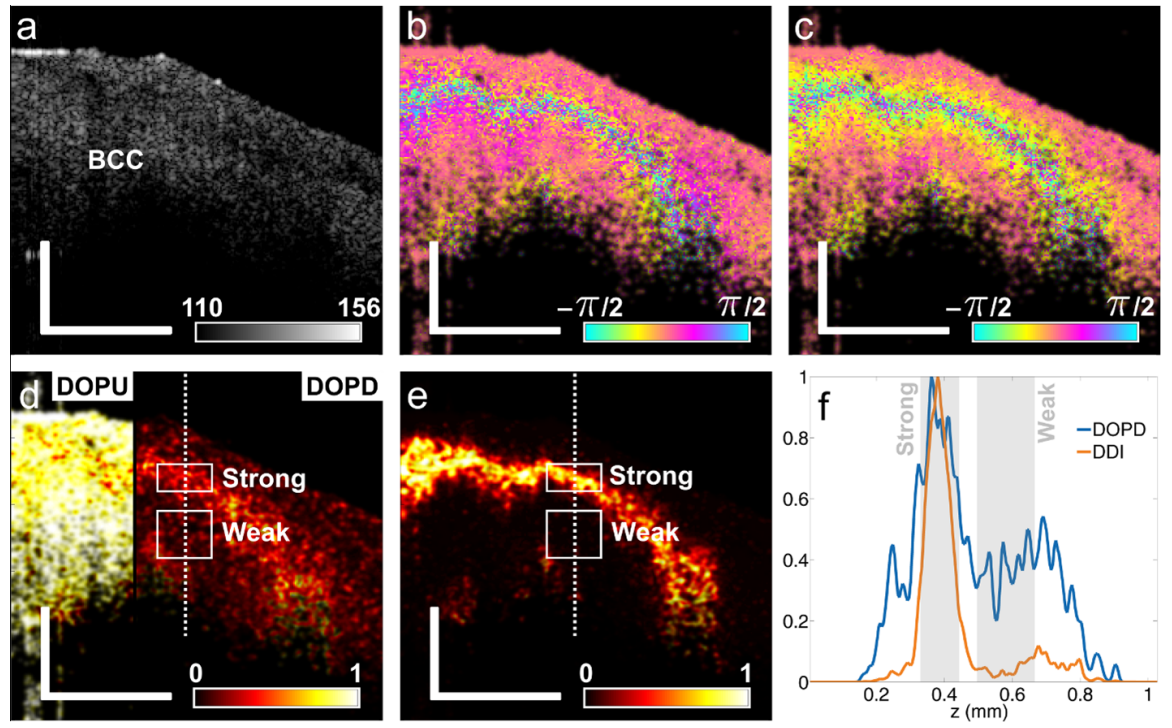

Figure 3. Human skin (a) reflectance (in $\mathrm{dB}$ ), where BCC indicates the basal cell carcinoma tumor position; linear retardance along the $x-y$ (b) and $\pm 45^{\circ}$ (c) directions (details are given in the text); (d) normalized DOPU (left) and DOPD (right); (e) normalized DDI; and (f) normalized DOPD and DDI along the dashed line shown in (d) and (e). The scale bar is $0.5(\mathrm{x}) \times 0.3(\mathrm{z}) \mathrm{mm}$. The relative contrast increase is $90.2 \%$. 

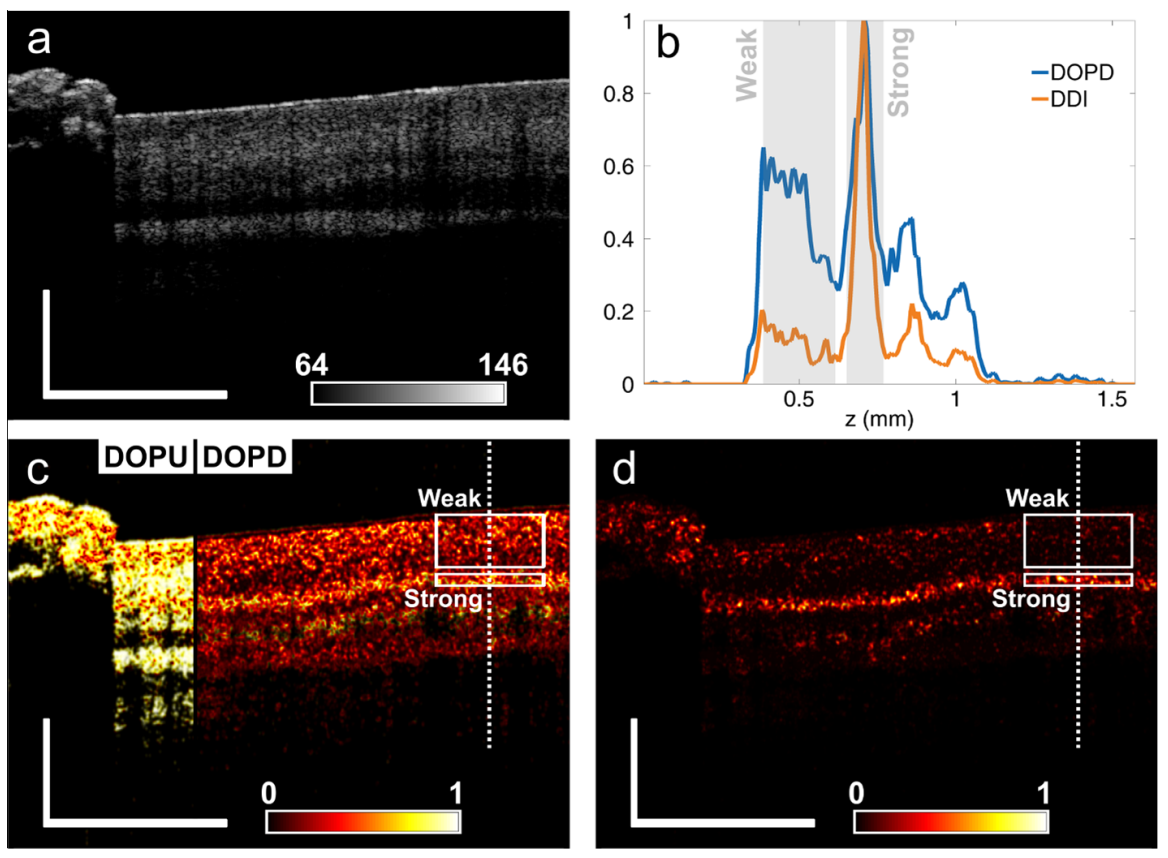

Figure 4. Human nail fold (a) reflectance (in dB); (c) normalized DOPU (left) and DOPD

(right); and (d) normalized DDI. The inset (b) depicts the normalized DOPD and DDI along the dashed line. Scale bar is $0.5 \times 0.5 \mathrm{~mm}$. The relative contrast increase for the background and ROI shown is $84.7 \%$. 